\title{
Synthesis of Carbon Nanofibers by Spray Pyrolysis
}

\author{
J.L. Bernal ${ }^{1}$, A. García-Barrientos ${ }^{1}$, A. Juanico ${ }^{2}$, A. Medina-Flores ${ }^{3}$, L. Béjar-Gómez ${ }^{3}$, J. solís ${ }^{4}$. \\ ${ }^{1 .}$ Universidad Politécnica de Pachuca. Carretera Pachuca-Cd. Sahagún, km 20, Ex-Hacienda de Santa \\ Bárbara Municipio de Zempoala, Hidalgo. \\ 2. Universidad Politécnica del Valle de México. Avenida Mexiquense s/n. C.P. 54910. \\ Tultitlán Estado de México. \\ 3. UMSNH. Ciudad Universitaria, Morelia, Michoacán. 58000. México. \\ 4. SEP-DGEST-IT de Tlalnepantla. Tlalnepantla de Baz, Edo de México. 54070.
}

Due to its special properties, carbon-related nanomaterials have attracted increasing interest among scientists around the world in both synthesis and applications. Nowadays, these nanomaterials represent one of the major research topics of science and technology of materials, as well as condensed matter physics [1-4]. Carbon nano-fibers (CNFs) are composed of stacked and curved graphene layers from a quasi-one-dimensional (1D) filament. CNFs have cylindrical or conical nanostructures. Their diameters vary from a few to hundred nanometers, while lengths vary from less than a micrometer to millimeters. As shown in Figure 1, according to the angle between graphene layers and fiber axis, the morphological structure is often divided into plate CNFs, ribbon-like CNFs, herringbone CNFs [5-7].

Carbon Nano fibers (CNFs) were synthesized by spray-pyrolysis in an ultrasonic nebulizer (Nebucor E$505,5 \mathrm{~W}$ ) connected to a quartz tube using hexane as a precursor. Silicon monocrystal substrates were placed at the center of the quartz tube. A flow of $\mathrm{N}_{2}$ gas was introduced into a quartz tube at constant rate of $15 \mathrm{ml} / \mathrm{min}$. Once a suitable reaction temperature of $800{ }^{\circ} \mathrm{C}$ was reached, the ultrasonic nebulizer was switched on, and the hexane nebulized was deposited into a thin film for 30 minutes. After deposition, the furnace was switched off and allowed to cool at room temperature. Finally, the deposited samples were analyzed by scanning electron microscopy (SEM) and energy dispersive spectroscopy (EDS) techniques. Figure 2a show (SEM) image of the CNFs cluster nano-fibers formed during the synthesis by spray-pyrolysis and figure 2c shows the chemical composition of the nano-fibres showing that all fibers are composed by carbon as main element, Fe and Si signals came from the reactor walls and organic precursor used. Figure $2 \mathrm{~b}$ shows a tubular filament solid CNF with a diameter ranking from $0.085 \mu \mathrm{m}$ to $0.114 \mu \mathrm{m}$. These results show that the Carbon Nanofibers (CNFs) can be synthesized by nebulized spray-pyrolysis in a single step process. CNFs can be formed from hexane at lower temperature and the repetition of the experiment resulted in a similar growth pattern of the deposits, demonstrating that conditions are suitable for CNFs growth.

References:

[1] J. Bao, et al. Materials Letters. Volume 68 (2012), p. 240-242.

[2] K. Awasthi, et al. Mater. Sci. Volume 34, No. 4, (2011), p. 607-614.

[3] K. Jong, J. Geus, Carbon Nanofibers. Catal. Rev. Volume 42 (2000), p. 481-510.

[4] K. Awasthi, et al. Mater. Sci. Volume 34, No. 4 (2011), p. 607-614.

[5] A. Mostofizadeh, et al. Journal of Nanomaterials Volume 2011, (2011), p 1-21.

[6] M. Endo, et al. J. Mater. Res. Volume 14 (1999), p. 4474-4477.

[7] S.Y. Gu, J. Ren, Q.L. Wu. Synth. Met. Volume 155 (2005), p. 157-161. 


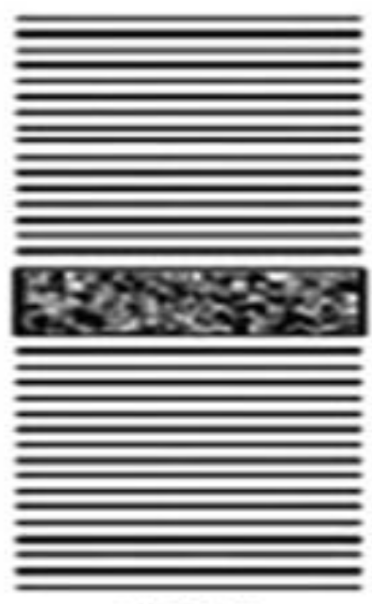

CNF-P

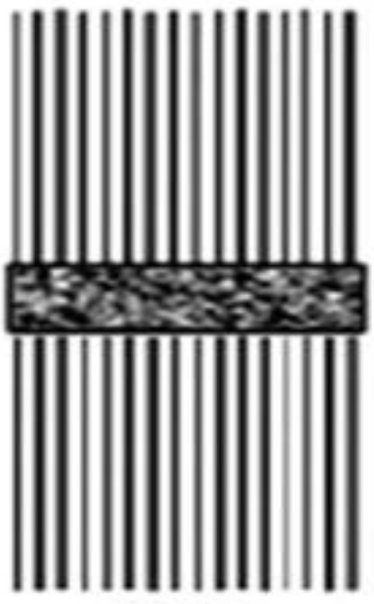

CNF-R

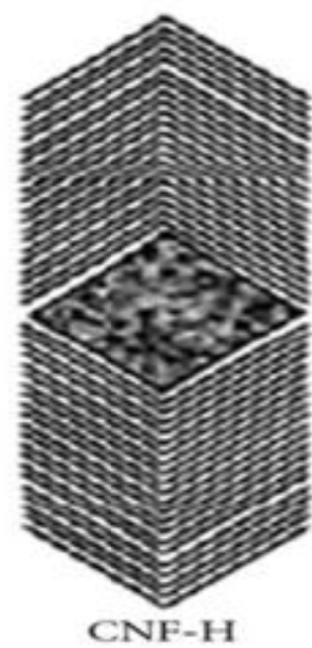

CNF-H

Figure 1. Schematic Diagram of different CNFs: Platet, Ribbon and Herringbon [5-10].
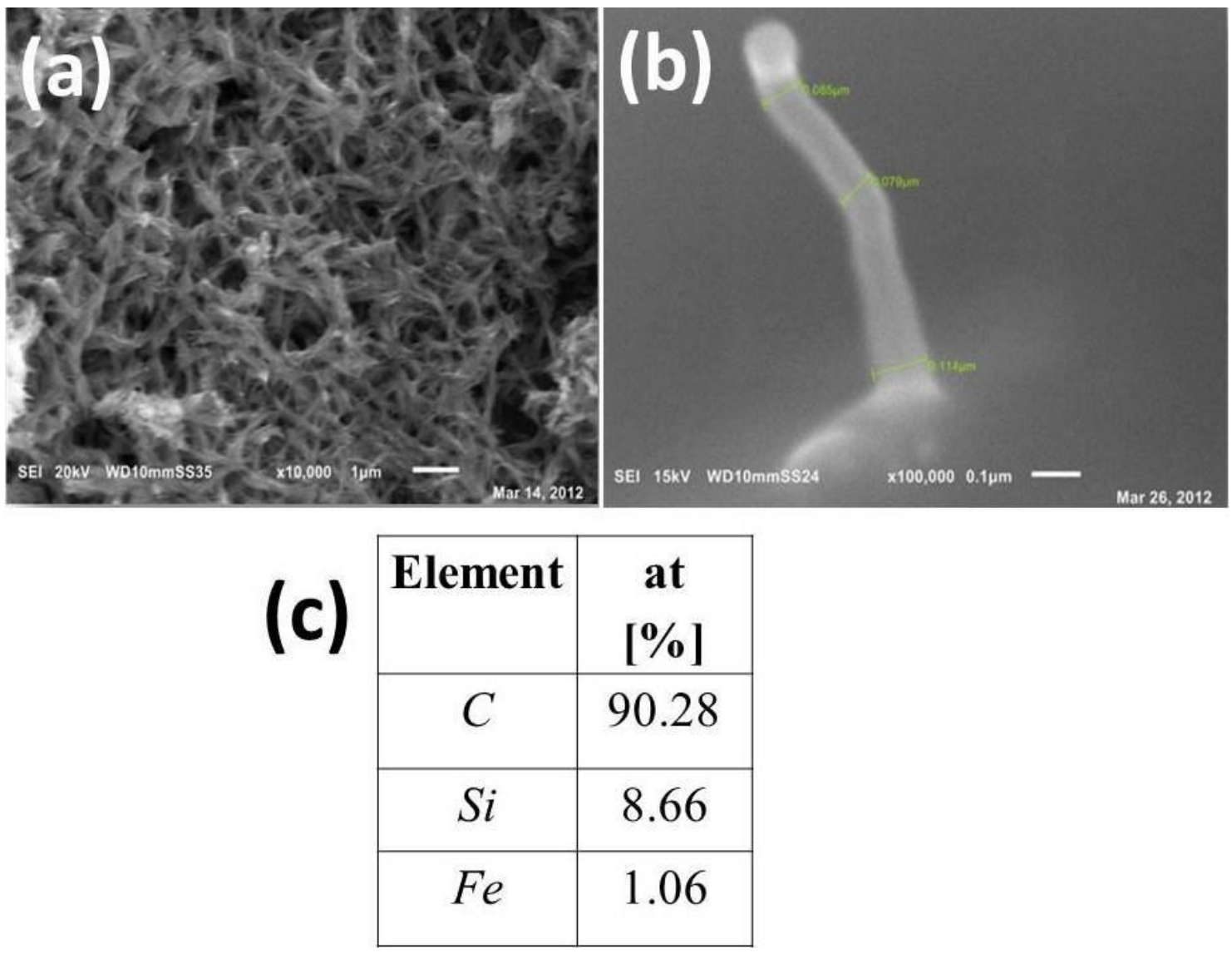

Figure 2. (a) SEM Image of CNF Cluster, (b) SEM Image of a Single CNF and (c) chemical composition of a Single CNF. 